\title{
Resistance to antimicrobials in humans and animals
}

\author{
Overusing antibiotics is not the only cause and reducing use is not the only solution
}

$\mathrm{W}$

arning signs of antimicrobial resistance, chinks in the antimicrobial armour, began to appear in the middle of the last century, and by the $1990 \mathrm{~s}$ various reports had signalled the dangers of excessive or inappropriate use of antibiotics in clinical medicine and of the use of antibiotics in animal feed as growth promoters. ${ }^{1-3}$ Overuse of antimicrobials emerged as the main culprit, and reducing their use was seen as the answer. But it may not be that simple.

The idea that reducing antibiotic use would redress the problem formed part of a positive response on the part of the United Kingdom government to the House of Lords report, ${ }^{1}$ including a public information campaign, surveillance of resistance along the food chain, targets with respect to hospital acquired infections, and setting up of an overarching advisory body on all aspects of antibiotic use. However, the concept of overuse has proved too simplistic, for, although the evidence of overprescribing as the main driver of resistance is overwhelming, ${ }^{4}$ reductions in antibiotic use have not necessarily resulted in lowered levels of resistance: some pathogens' resistance has remained stable, and with others it may even have increased. For example, although penicillin resistance in Streptococcus pneumoniae has been declining, ${ }^{5}$ macrolide resistance in $S$ pneumoniae has remained stable, ${ }^{6}$ and other species such as Haemophilus influenzae have shown no evidence of a fall in resistance.

Resistance to fluoroquinolones in Escherichia coli has increased sharply despite a reduction in prescribing. ${ }^{8}$ Such resistant strains are thought to originate from patients' gut flora. ${ }^{4}$ The advent of extended spectrum $\beta$ lactamases in $E$ coli isolates resistant to cephalosporins through CTX-M enzymes now pose problems with urinary tract infections being resistant to the fluoroquinolones. The relation of $E$ coli with extended spectrum $\beta$ lactamases in animals to human infection is not yet clear. Such $E$ coli can cause severe diarrhoea and death in calves, but it also has the ability to confer resistance to a wide range of $\beta$ lactam compounds such as penicillin and cephalosporins.

Reports of hospital infections with methicillin resistant Staphylococcus aureus (MRSA) have epitomised antibiotic resistance in the public eye and galvanised hospital authorities to improve ward cleanliness and hygiene. MRSA infection is most prevalent in surgical wards and in long term care facilities, where indwelling devices are used, and has recently been recognised as a community problem in the United States. ${ }^{9}$ The organism is commensal on humans under ordinary conditions, and varying percentages of nursing staff have been found to carry the organism. ${ }^{10}$ It is also found on the coats of animals, and in animal surgery has accounted for the same problems in dogs, horses, and a cat as it has in human surgery. Transfer of resistant organisms between companion animals and human patients has been detected, ${ }^{11}$ and it is generally derived from humans. MRSA has not been reported from food animals.

The use of antibiotics in animals has raised controversy, particularly with respect to their use as feed additives to promote growth of livestock. There can be little doubt that the clinical therapeutic use of antibiotics in animals is justified, and, with large collections of animals, individual treatment is not feasible-hence drug delivery in water or feed is necessary and justified. However, the difference between mass prophylaxis and growth promotion is poorly defined. For example, the fluoroquinolone enrofloxacin is used to treat respiratory infections in turkeys and chickens and is sometimes given to an entire flock in water. The concern is that the use of enrofloxacin leads to drug resistant Campylobacter-a common cause of food poisoning in humans-and the prevalence of fluoroquinolone resistant Campylobacter in humans is increasing. ${ }^{12}$ As a result, the US Food and Drug Administration has recently banned enrofloxacin's use in poultry (July 2005).

Certain growth promoters have also been banned in the European Union, but controversy continues as to the potential health risk of transfer of antibiotic resistance from animals to man when set against the increased production costs and food prices-a trade off between public health and economic benefits. The British veterinary profession and livestock community have responded well to the challenge of potential antibiotic resistance in animals. RUMA (Responsible use of Medicines in Agriculture Alliance), a consortium of 18 organisations, has addressed the use of antibiotics in the main species of farm animal and has recently launched guidelines on the "responsible use of antimicrobials" in these species, with the comment that human health must be the overriding consideration. This is a unique initiative involving organisations representing every stage of the process from "farm to fork" and promoting the highest standards of food safety and animal health and welfare.

The magic bullets have lost some of their magic. One solution may be to find alternatives to antibiotics when resistance appears, but there is also an urgent need for new antibiotics to be developed. Few pharmaceutical companies are now involved in antibiotic development, however. The high cost of development, the prolonged safety evaluation, and the probable short duration of field use and the present tendencyfor any new compound to induce resistance all militate against major investment in new compounds.

Lord Soulsby of Swaffham Prior emeritus professor of animal pathology and member of the Specialist Advisory Committee on Antimicrobial Resistance (SACAR)

University of Cambridge, Cambridge

(lordsoulsby@swaffham-prior.co.uk)

Competing interests: None declared.

Resistance to antibiotics and other antimicrobial agents. Report of the House of Lords Select Committee on Science and Technology. London: Stationery Office, 1998. 
2 Standing Medical Advisory Committee: Sub-Group on Antimicrobial Resistance The path of least resistance. London: Department of Health, 1998 World Health Organization. The medical impact of the use of antimicrobials in food animals: Report of a WHO meeting, Berlin, Germany 13-17 October 1997. Geneva: WHO, 1997.

Livermore DM. Minimising antibiotic resistance. Lancet Infect Dis 2005;5:450-9.

Livermore DM. Can better prescribing turn the tide of resistance? Nat Rev Microbiol 2004;2:73-8.

Wise R. The relentless rise of resistance? J Antimicrob Chemother 2004;54: 306-10.

7 Reynolds R, Shackcloth J, Felmingham D, MacGowan A. Antimicrobial susceptibility of lower respiratory tract pathogens in Great Britain and susceptibility of lower respiratory tract pathogens in Great Britain and
Ireland 1999-2001 related to demographic and geographic factors: the BSAC respiratory resistance surveillance programme.J Antimicrob Chemother 2003;52:931-43.
8 Livermore DM, James D, Reacher M, Graham C, Nichols T, Stephens P, et al. Trends in fluoroquinolone (ciprofloxacin) resistance in enterobacteraceae from bacteremias, England and Wales, 1990-1999. Emerg Infect Dis $2002 ; 8: 473-8$.

Naimi TS, LeDell KH, Boxrud DJ, Groom AV, Steward CD, Johnson SK, et al. Epidemiology and clonality of community-acquired methicillinresistant Staphylococcus aureus in Minnesota, 1996-1998. Clin Infect Dis 2001;33:990-6.

10 Scott GM, Thomson R, Malone-Lee J, Ridgway GL. Cross-infection between animals and man: possible feline transmission of Staphylococcus aureus infection in humans? J Hosp Infect 1988;12:29-34.

11 Weese JS, Archambault M, Willey BM, Hearn P, Kreiswirth BN, Said-Salim B, et al. Methicillin-resistant Staphylococcus aureus in horses and horse personnel, 2000-2002. Emerg Infect Dis 2005;11:430-5.

12 Piddock LJ. Quinolone resistance and Campylobacter spp. J Antimicrob Chemother 1995;36:891-8.

\title{
What could Dr Finlay and Mr Herriot learn from each other?
}

\author{
Comparisons of human and animal diseases can benefit patients of all species
}

$\mathrm{T}$ The late 19th century brought recognition that normality and disease depended on similar mechanisms in humans and animals; leading scientists espoused the concept of "one medicine," calling it the medicine of the future. Comparative medicine largely failed to realise these expectations during the 20th century, becoming narrowly equated with induced rodent models of human disease. But veterinary and medical research have plenty to learn from each other.

Comparative medicine is the study of comparable diseases in different species; similarities and differences are both informative. Induced models are useful for identifying potential disease mechanisms, but spontaneous models come into their own with multifactorial diseases combining genetic, dietary, environmental, toxic, immune, and other factors-which are inherently difficult to model-and for the assessment of new treatments ahead of human trials, thereby bringing veterinary patients faster access to clinical innovations.

For many diseases-such as asthma, cystic fibrosis, and various cancers-rodent models are misleading, and therapeutic responses may differ profoundly from

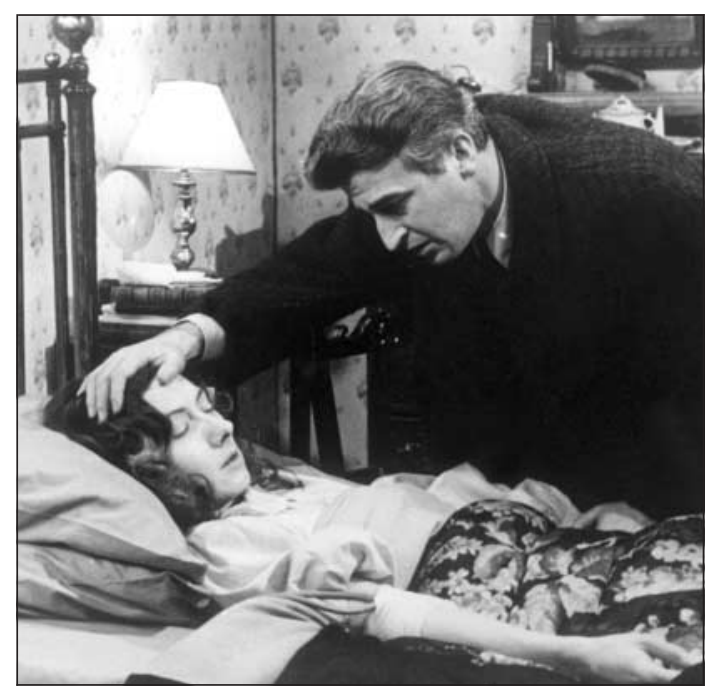

The iconic television general practitioner, Dr Finlay humans. ${ }^{1}$ The dog, for example, has several advantages over the mouse as a model of human disease. ${ }^{2}$ Many human tumours have canine counterparts, ${ }^{3}$ and, unlike in mice, telomerase reactivation may herald the onset of malignancy in dogs, potentially offering a therapeutic target. ${ }^{4}$

Our understanding of human hypertension is a good example of the power of comparative research. Knowledge of hypertension predated clinical blood pressure measurements; salt and renal disease were implicated 3000 years ago. The earliest blood pressure measurements, by Hales in 1733, mainly relied on dogs, as did the "Goldblatt model" of hypertension 100 years later and the research underlying Guyton's concept of "pressure natriuresis" as the long term regulator of arterial pressure. Genetic and salt sensitive hypertension and the renal role in blood pressure regulation and in renovascular and other forms of hypertension all emerged from studies in animals, notably dogs. Differences between breeds of dog in arterial pressure may yet offer insights into mechanisms or evolutionary origins of human essential hypertension: did the same genes confer biological advantage when human life was shorter and more active?

Lack of a comparative perspective often flaws research on hypertension through the use of exorbitant sodium intakes-such as rats given a diet comprising $8 \%$ salt, equivalent to a human consuming $6400 \mathrm{mmol} /$ day (a month's customary intake daily). Actually, mammalian requirement is probably below $0.6 \mathrm{mmol} / \mathrm{kg} / \mathrm{day}$, so the rat diet represents five months' nutritional requirement daily. ${ }^{5}$ The benefits of comparative research, exemplified by hypertension, include control over factors difficult to control in humans (such as diet and breeding), short generation time, and plentiful siblings (invaluable for studies of prenatal or perinatal factors in disease), and shorter lifespan and breed differences in lifespan (opportunities to study diseases of senescence and the biological basis of ageing).

Comparative medicine comes into its own in the field of genetics-genes shared between species far outnumber those that are unique. Alignment of 